\title{
Optimizing Legacy Molecular Dynamics Software with Directive-Based Offload
}

\author{
W. Michael Brown ${ }^{\mathrm{a}, *}$, Jan-Michael Y. Carrillo ${ }^{\mathrm{b}}$, Nitin Gavhane ${ }^{\mathrm{c}}$, Foram M. Thakkar ${ }^{\mathrm{d}}$, Steven J. Plimpton \\ ${ }^{a}$ Intel Corporation, Portland, Oregon, USA \\ ${ }^{b}$ Center for Nanophase Materials Sciences and Computer Science and Mathematics Division, Oak Ridge National Laboratory, Oak Ridge, Tennessee, USA \\ ${ }^{c}$ Computational Centre of Expertise, Shell India Markets Private Limited, Bangalore, India \\ ${ }^{d}$ Computational Centre of Expertise, Shell India Markets Private Limited, Bangalore, India \\ ${ }^{e}$ Multiscale Science, Sandia National Laboratories, Albuquerque, NM, USA
}

\begin{abstract}
Directive-based programming models are one solution for exploiting many-core coprocessors to increase simulation rates in molecular dynamics. They offer the potential to reduce code complexity with offload models that can selectively target computations to run on the CPU, the coprocessor, or both. In this paper, we describe modifications to the LAMMPS molecular dynamics code to enable concurrent calculations on a CPU and coprocessor. We demonstrate that standard molecular dynamics algorithms can run efficiently on both the CPU and a x86-based coprocessor using the same subroutines. As a consequence, we demonstrate that code optimizations for the coprocessor also result in speedups on the CPU; in extreme cases up to 4.7X. We provide results for LAMMPS benchmarks and for production molecular dynamics simulations using the Stampede hybrid supercomputer with both Intel ${ }^{\circledR}$ Xeon Phi ${ }^{\mathrm{TM}}$ coprocessors and Nvidia GPUs. The optimizations presented have increased simulation rates by over $2 \mathrm{X}$ for organic molecules and over $7 \mathrm{X}$ for liquid crystals on Stampede. The optimizations are available as part of the "Intel package" supplied with LAMMPS.
\end{abstract}

Keywords:

Molecular dynamics, Xeon Phi, GPU, coprocessor, accelerator, many-core

\section{Introduction}

Issues with power consumption and heat dissipation have led to a trend towards many-core processors as an approach to increase parallelism with electrical power efficiency. Although bootable many-core processors are expected to be available in the future, current high performance computers exploit manycore processors with a hybrid configuration using nodes containing traditional CPUs along with graphics processing units (GPUs) or Intel ${ }^{\circledR}$ coprocessors. In these designs, source code

\footnotetext{
${ }^{*}$ Corresponding author.

URL: michael.w.brown@intel.com (W. Michael Brown),

carrillojy@ornl.gov (Jan-Michael Y. Carrillo),

Nitin.Gavhane@shell. com (Nitin Gavhane),

Foram.Thakkar@shell.com (Foram M. Thakkar), sjplimp@sandia.gov (Steven J. Plimpton)
}

modifications are necessary in order to efficiently use the system.

In our previous work, we have focused on the design of efficient algorithms to use GPU accelerators for large-scale molecular dynamics (MD) simulations [1, 2, 3, 4]. For this work, a separate library was designed for the LAMMPS molecular dynamics software [5] with MD algorithms modified to run efficiently on GPUs. This library could be compiled using either CUDA or OpenCL. Although this approach has allowed for GPU-acceleration in production simulations, the use of a separate programming language and different algorithms on the CPU and GPU introduces additional code complexity and requires optimization of separate code paths depending on the target. For example, in MD, redundant calculation is typically used to avoid memory conflicts for force updates on the GPU 
where greater than 10000 threads can be in flight simultaneously. In the case of 3 -body potentials, this requires up to 3 times as many force calculations compared to the CPU algorithm, new neighbor list routines, and doubling of the volume of the border regions between neighboring MPI tasks in the spatial decomposition [4].

The Intel ${ }^{\circledR}$ Xeon Phi ${ }^{\mathrm{TM}}$ coprocessor is an x86-based manycore processor that also connects to the host through the PCI express bus. Because the coprocessor runs a full-service Linux operating system, there are several options for using the coprocessor in HPC systems. These include "native" mode, where code is run solely on the coprocessors without involving the host processor, "symmetric" mode, where MPI tasks run on both the CPUs and the coprocessor, and "offload" mode, where the host offloads some of the work to be performed on the coprocessor. The best choice will depend upon a number of factors, but for legacy HPC software, "offload" provides some advantages in that optimizations can be focused on select compute-intensive routines without consideration or alteration of the distributed memory parallelization. Therefore, we chose this approach to evaluate modification to the LAMMPS MD software to allow for utilization of Intel ${ }^{\circledR}$ Xeon $\mathrm{Phi}^{\mathrm{TM}}$ coprocessors. Because the architecture is $\mathrm{x} 86$-based, we also examine how the modifications influence performance on more traditional Intel ${ }^{\circledR}$ Xeon $^{\circledR}$ CPUs.

\section{Methods}

\subsection{LAMMPS}

In this work, we are considering enhancements to the LAMMPS molecular dynamics package [5]. LAMMPS is parallelized via MPI, using spatial-decomposition techniques that partition the simulation domain into smaller subdomains, one per processor. In this approach, each MPI task owns a set of local atoms that are within the subdomain, but also stores data for ghost atoms that are owned by another MPI task and within the cutoff distance at the subdomain borders. For these atoms at the borders, MPI communication can occur every timestep because their energy is affected by atoms owned by multiple MPI tasks.
LAMMPS is a general purpose MD code capable of simulating biomolecules, polymers, materials, and mesoscale systems. It is also designed in a modular fashion with the goal of allowing additional functionality to be easily added. This is achieved via a variety of different style choices that are specified by the user in an input script and control the choice of force-field, constraints, time integration options, diagnostic computations, etc. At a high level, each style is implemented in the code as a $\mathrm{C}++$ virtual base class with an appropriate interface to the rest of the code. For example, the choice of pair style (e.g. lj/cut for Lennard-Jones with a cutoff) selects a pairwise interaction model that is used for force, energy, and virial calculations. Individual pair styles are child classes that inherit the base class interface. Thus, adding a new pair style to the code (e.g. lj/cut/hybrid for a Lennard-Jones potential optimized for hybrid execution, is as conceptually simple as writing a new class with the appropriate handful of required methods or functions, some of which may be inherited from the parent-class pair style (lj/cut in this case).

\subsection{Offload}

For this work, we have used the Intel ${ }^{\circledR}$ Language Extensions for Offload (LEO) directives to handle data allocation on the coprocessor, asynchronous data transfer and computation offload, and synchronization. Because directives are used, the code can be compiled by any $\mathrm{C}++$ compiler and used on machines that do not contain coprocessors. Additionally, LEO supports an if clause allowing the same routine to be called with and without offload. This can be used to perform computations on the CPUs and the coprocessor simultaneously. The LEO model is advantageous in that the code inside an offloaded region supports $\mathrm{C}++$ and Fortran, has no restrictions on function calls to other routines, and can use different parallel programming models such as OpenMP, POSIX Threads, or Intel ${ }^{\circledR}$ Cilk $^{\mathrm{TM}}$ Plus. Example directives with LEO are given in the listing:

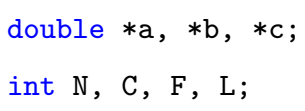




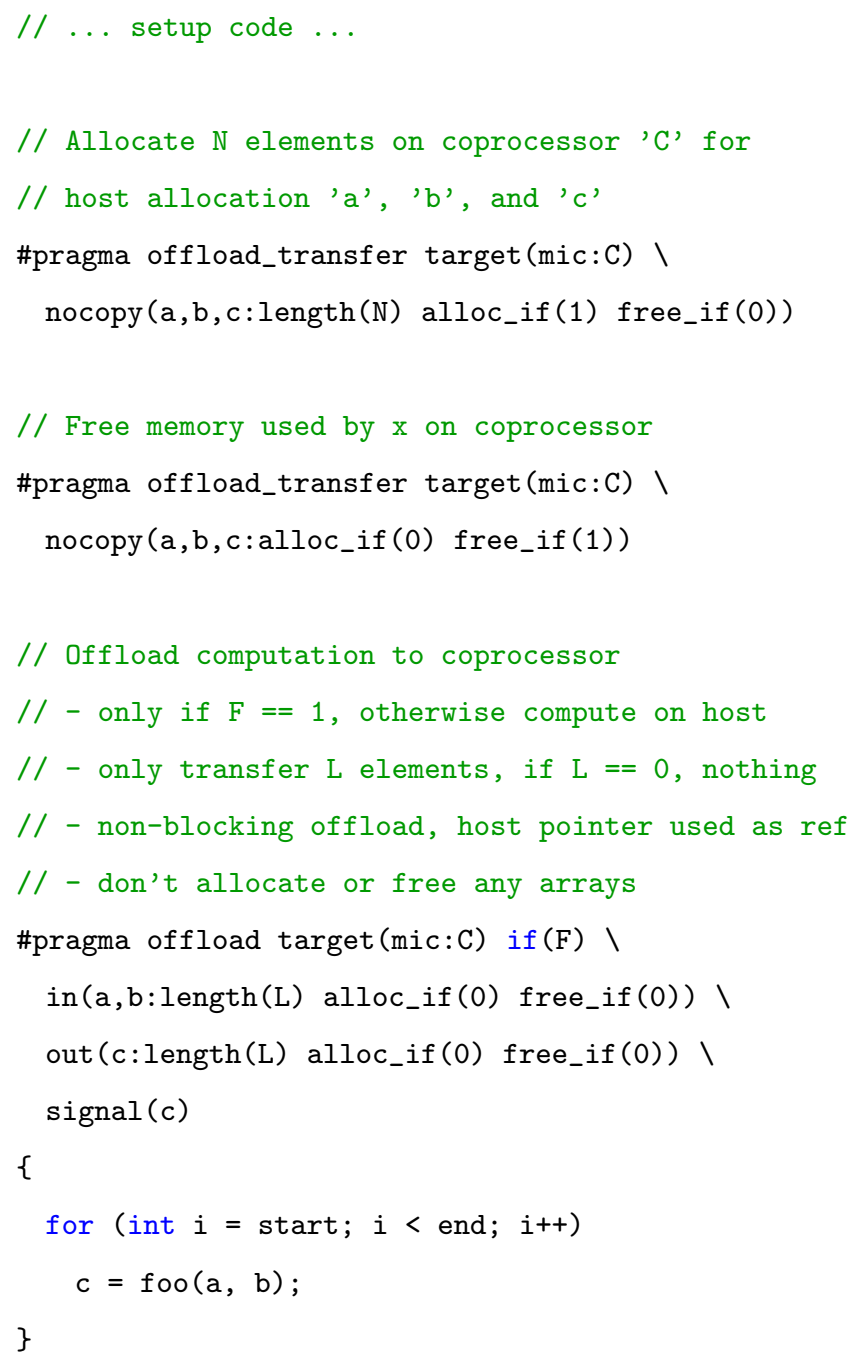

Offload is used for both neighbor list builds and calculation of short-range terms including energies, forces, torques, and virials. In order to obtain performance, memory allocation on the coprocessor is never performed within a loop, unless necessary to grow an allocation to fit needed data. Additionally, data transfer for constant quantities (atom types, charges, etc.) is only repeated on timesteps when neighbor lists are re-built, since those are the only steps when atoms migrate to new processors, changing the per-atom data structures.

In order to utilize both the CPU and coprocessor on hybrid machines, offload for a fraction of the work is supported. The fraction is supplied as an optional parameter for the simula- tion run. If the offload fraction is 0 , the coprocessor is not used. In the default case, dynamic load balancing is performed to automatically adjust the fraction at each neighbor list build based on the computational time being monitored on both the host and coprocessor. The objective is to minimize idle time on both. Currently, we are unable to attain data transfer times using LEO and therefore they are not included in the load balance calculations. The fraction for load balancing is calculated using a weighted mean with a 0.1 weight for the current time step and 0.9 for the previous value. The load balance fraction is calculated so as to balance the time for neighbor list and short-range calculations on the coprocessor versus neighbor list, short-range, long-range, and bond/angle calculations on the host, since they run concurrently.

We have implemented two different approaches for dividing the work between the host and the coprocessor. In the first, the local atoms are divided so that the host and coprocessor each loop over the neighbors of a distinct set of atoms. In the second, the coprocessor is still assigned a subset of the local atoms, but no ghost atoms are included in the neighbor lists used on the coprocessor. The host loops over all local atoms, but for those evaluated on the coprocessor, only ghost atoms are included in the neighbor list. This approach offers more flexibility in overlapping MPI communications with computation on the coprocessor. In both cases, neighbor list builds keep track of the minimum and maximum indices for atoms used in the lists. Because LAMMPS employs methods to sort atom data based on spatial location, this approach can be used to reduce the amount of data transfer with the coprocessor and also the range of atoms involved in force accumulation for thread-private arrays.

In order to allow for an arbitrary configuration of MPI tasks and OpenMP threads on the host, multiple MPI tasks are allowed to offload work to available coprocessors. On each node, the MPI tasks are divided into groups, with one group for each coprocessor on the node. For each coprocessor, the available threads are divided evenly among the group of MPI tasks. Each MPI task gets exclusive affinity for its threads by using the $k m p \_s e t \_a f f i n i t y \_m a s k \_p r o c$ function. For example, if each 
node has a single coprocessor with 60 cores and 240 hardware threads available for offload, running 24 MPI tasks per node would allocate 10 coprocessor threads to each MPI task.

\subsection{Vectorization}

As vector widths have continued to increase, exploiting vectorization in the software has become increasingly important for power-efficiency. On the Intel ${ }^{\circledR}$ Xeon Phi ${ }^{\mathrm{TM}}$ coprocessor, the vector width is 512 bits. Although the compiler can automatically vectorize loops, it is sometimes necessary to make changes to the software in order to tell the compiler that vectorization is safe or to improve vectorization efficiency. In the case of the GPU algorithms used in LAMMPS, parallelization occurs over both the outer loop (local atoms) and the inner loop (neighbors of local atoms). Since the same neighbor can occur in the neighbor list of multiple local atoms, there is potential for collisions where multiple threads attempt to simultaneously update the same memory location. This is typically handled by ignoring Newton's third law and computing the force $F_{i j}$ between atoms $I$ and $J$ twice, once by the thread for atom $I$ and once by the thread for atom $J$. In LAMMPS, this can be invoked by doubling the memory used for the neighbor list (for 2-body interactions); atom $J$ appears in atom $I$ 's list of neighbors, and vice versa. An alternative strategy, if deterministic algorithms are not required, is to use atomic operations or locks for the memory updates.

For the Intel ${ }^{\circledR}$ Xeon Phi ${ }^{\mathrm{TM}}$ coprocessor, other options are feasible. Because the number of threads in flight is orders of magnitude lower, inner-loop vectorization can be used efficiently and outer-loop parallelization can be achieved with data privatization where every thread has a different copy of a force array for the necessary atoms. This approach requires summing all copies of atom forces into a single array at the end of the calculation. In this case, the same algorithms can be used on the host and the coprocessor. This is the approach that we have implemented here. Because the compiler does not know that an atom index will only occur once within the neighbor list of another atom, it is necessary to instruct the compiler that the inner loop is safe to vectorize. Additionally, on timesteps where the energy or pressure is needed, pairwise energy and virial terms must be accumulated into a single variable. Both of these issues can be addressed by placing “\#pragma simd reduction(+:.)" before the inner loop where '.' is a comma-delimited list of variables to be accumulated at the end of the loop.

The use of single and mixed precision calculations is known to improve performance in molecular dynamics. Storing data in single precision can reduce random-access memory latencies and single precision calculations have twice the number of data lanes for vector computations (and sometimes more on GPU hardware). Additionally, the Intel ${ }^{\circledR}$ Xeon Phi ${ }^{\mathrm{TM}}$ coprocessor includes hardware support for fast transcendentals in single precision. To support single and mixed precision, we have added new memory allocations for atom data. Although this approach requires packing and (possibly) casting atom data every timestep, it allows for full compatibility with existing LAMMPS routines that use the double-precision versions, without modifying data structures that are used throughout half a million lines of code. Because we are using new structures, we have also changed the atom data to be aligned to 4-element boundaries for each atom. In the case of position data, the atom type is stored along with the position (e.g. $x_{1} y_{1} z_{1}$ type $_{1}, x_{2}$ $y_{2} z_{2}$ type $\left._{2}, \ldots\right)$. For forces and torques, the 4 th element is unused. This alignment is advantageous in that it can improve vectorization performance when using Intel ${ }^{\circledR}$ Advanced Vector Extensions (AVX) (where gather instructions are not used), ensures that data for a single atom will be on a single cache line, and reduces cache misses for random access to atom type data.

In order to perform vector computations for the force calculation, the data for the atoms in the neighbor list must be gathered into vector registers. These gather operation can reduce the vectorization intensity. Approaches to mitigate this effect include the use of explicit vector intrinsics to perform fast gathers for atom data [6] and approaches to build neighbor lists using groups of atoms with data packed to improve vector performance for each group [7]. Due to the fact that the vector intrinsics are architecture and floating-point precision specific, 
we have chosen to omit this optimization in favor of portable code with reduced complexity. For the same reasons, we have not explored neighbor groups, due to the large variance in typical neighbor list sizes for the many simulation models available in LAMMPS. We note that both optimizations have the potential to improve performance further for the measurements presented here, however, and that new features in version 15 of the Intel ${ }^{\circledR}$ compilers can potentially be exploited for explicit vectorization of only the atom data gather.

One other optimization to improve vectorization efficiency was required for the coarse-grained, orientation-dependent Gay-Berne potential [8], commonly used to model ellipsoidal particles. If the number of neighbors is not a multiple of the vector width, different instructions can be used to handle the "loop remainder" in order to prevent out-of-bounds memory access along with other issues. For the Gay-Berne potential, the number of instructions in the vectorized force calculation is very large due to the complexity of the anisotropic potential. In this case, a significant amount of time was found to be in the remainder code. For this reason, we pad the neighbor list (for all potentials) when needed, with an index to a dummy atom that is guaranteed to never be within the cutoff. This forces the number of neighbors for each atom to be a multiple of the vector width, so that remainder code is never executed.

\subsection{Data Alignment}

One other optimization that proved to be important is that all data allocations on the heap were aligned to 64B boundaries along with important data allocations on the stack. This is important for several reasons. Data transfer between host memory and the coprocessor is faster for aligned data. Vectorization performance is better for aligned data. Finally, this alignment eliminates false sharing for persistent data allocations used for per-thread arrays. This optimization was trivial in LAMMPS; a preprocessor define was set to force alignment on the heap and an additional alignment qualifier was added to a few allocations on the stack.

\subsection{GPU Comparison}

At a high level, the work presented here is similar to our previous GPU implementation for LAMMPS [1, 2], in that offload of neighbor list builds and short-range calculations are run concurrently with other calculations on the host. While the GPU implementation supports offload of routines for the particleparticle particle-mesh solver, on modern hardware there is often little or no benefit from this. Optimizing these routines with vectorization on the host CPUs will almost certainly improve performance, however, this was not performed as part of this work. One important difference from the GPU implementation is that the same routines can run on the host processor, even if there is no coprocessor on the system. The advantage of this will be discussed below.

Additionally, there are differences in how the GPU or coprocessor are shared between multiple MPI tasks. For the GPU, CUDA Multi-Process Service (MPS) is used; in this case the number of MPI tasks sharing the GPU is limited to 16 and there is no control over how the kernels from different MPI tasks are scheduled on the GPU multiprocessors. If each kernel is run on the entire GPU, there is a potential advantage due to a natural pipelining that can overlap PCI express data transfers from some MPI tasks with computations from others. When running with few atoms per node, which is often the case on HPC clusters to increase simulation rates, using the entire GPU for each kernel can hurt performance. For the implementation presented here, the number of MPI tasks sharing the coprocessor is not limited and each MPI task has exclusive affinity for hardware threads on the coprocessor.

\subsection{Performance Measurements}

For the performance measurements here, the Jul 292014 version of LAMMPS was used with the modifications described in this paper. These modifications are now available as the "Intel Package" distributed with the current version of LAMMPS. The Stampede supercomputer [9] was used for the benchmarks. Stampede nodes have two Intel ${ }^{\circledR}$ Xeon ${ }^{\circledR}$ E5-2680 CPUs with turbo enabled and hyperthreading off (16 cores/16 threads per 
node). Each node has 32 GB DDR3 1600 memory. Most Stampede nodes have an Intel ${ }^{\circledR}$ Xeon $\mathrm{Phi}^{\mathrm{TM}} \mathrm{SE} 10 \mathrm{P}$ coprocessor with 61 cores running $1.1 \mathrm{Ghz}$, although some contain an NVIDIA Tesla K20m GPU. The Intel ${ }^{\circledR}$ coprocessors were running MPSS version 3.3 and configured with ECC on and turbo off. The Tesla K20m nodes were using the CUDA 6.0 driver (331.67) and were also configured with ECC on and turbo off. Stampede uses Mellanox FDR Infiniband in a fat tree configuration for the interconnect. The operating system was CentOS release 6.5.

Software was compiled using the Intel ${ }^{\circledR} \mathrm{C}++$ Compiler 2013 (version 14.0.1). The compiler flags used were “-g -O3 -xAVX -fno-alias -ansi-alias restrict -DLAMMPS_MEMALIGN=64 -overridelimits -offload-option,mic,compiler,“-fp-model fast=2 mGLOB_default_function_attrs=“gather_scatter_loop_unroll=4"'”, For the GPU library in LAMMPS, CUDA 5.5 was used with compiler flags " $-\operatorname{arch}=\mathrm{sm} \_35$-DUNIX $-\mathrm{O} 3 \quad$-Xptxas -V use_fast_math". MVAPICH2/2.0 $b$ was used as the MPI library and LAMMPS was built to use the Intel ${ }^{\circledR}$ Math Kernel Library with single precision FFTs.

For each node count and each workload, multiple runs were performed, each with a different configuration of MPI tasks and OpenMP threads. The MPI/OpenMP configuration resulting in the best time is reported. For runs offloading to a Intel ${ }^{\circledR}$ coprocessor, the number of MPI tasks and OpenMP threads were varied. Runs were performed using both dynamic load balancing and offload of a fixed fraction of work varied in intervals of 0.05. For runs offloading to a GPU, CUDA MPS was used as runs were performed using 1, 2, 4, 6, 8, 10, 12, 14, and 16 MPI tasks per node with the best performing run reported. Runs offloading to the GPU were performed with and without offload of particle-particle particle-mesh routines and the best time was taken.

The GPU library was compiled for mixed precision and all performance numbers reported here are from mixed precision runs aside from the unmodified LAMMPS performance running on only CPUs, which use double precision.

\section{Results}

We have evaluated several workloads for performance including protein and liquid crystal benchmarks, production simulations used to study molecular alignment in organic solar cells, and production simulations used to study the thermodynamic and transport properties of complex hydrocarbon mixtures. These workloads are described in more detail in the following text.

\subsection{Rhodopsin protein in solvated lipid bilayer}

The rhodopsin protein benchmark is one of the standard benchmarks provided with LAMMPS and therefore the easiest to reproduce. This is an all-atom benchmark simulating the rhodopsin protein in a solvated lipid bilayer using the CHARMM force field [10], particle-particle particle-mesh long-range coulombics [11], and SHAKE constraints [12]. The benchmark is based on simulations by Crozier et al. [13]. The model contains counter-ions and a reduced amount of water to make a 32000 atom system. An inner cutoff of $8 \AA$ and an outer cutoff of $10 \AA$ are used for short-range force calculations. The simulations are performed using the isothermal-isobaric (NPT) ensemble with a timestep of 2.0 femtoseconds. The benchmark was run for 100 timesteps on 1 to 32 nodes, following a 10 timestep warm-up run. The purpose of the warm-up run is to allow the load-balancer that divides the work between the CPU and coprocessor time to converge before starting the performance measurement. In order to show strong-scaling results for a larger simulation that is easily reproduced, we have replicated the simulation box to give a simulation size of $512 \mathrm{k}$ atoms. This is similar to our previous report using GPUs [2].

We define the "baseline" code as the LAMMPS code providing the best performance on CPUs prior to the optimizations presented in this paper and all speedups are compared to the baseline performance at a similar node count. For the rhodopsin benchmark on a single node, we find that the optimizations provide a $1.21 \mathrm{X}$ speedup when running only on the CPUs. In both cases, best performance was with a single OpenMP thread and 16 MPI tasks. For offload to GPU, the 
best performance is with 14 MPI tasks sharing the GPU and the resulting speedup is $2.3 \mathrm{X}$. Offload of a fixed fraction to the Intel ${ }^{\circledR}$ Xeon Phi ${ }^{\mathrm{TM}}$ coprocessor gives similar performance with a speedup of $2.16 \mathrm{X}$ with $75 \%$ of the atoms offloaded, including ghosts. If ghost atoms are excluded from offload, best performance is with $90 \%$ of atoms offloaded and the resulting speedup is $2.09 \mathrm{X}$. Using automatic load-balancing, we find that an average of $78 \%$ of atoms are offloaded across MPI tasks with a performance of $1.95 \mathrm{X}$ of the baseline code. In all single-node cases with offload to the Intel ${ }^{\circledR}$ coprocessor, best performance was with 16 MPI tasks and a single OpenMP thread. For multinode simulations, we find that the performance with the Intel ${ }^{\circledR}$ Xeon $\mathrm{Phi}^{\mathrm{TM}}$ coprocessor is still significantly improved down to 1000 atoms per CPU core with a speedup of $1.35 \mathrm{X}$ using 4 MPI tasks with 2 OpenMP threads each. This is in contrast to the GPU-accelerated runs which are slightly slower than the baseline at 32 nodes $(0.99 \mathrm{X})$. For offload to 32 Intel $^{\circledR}$ coprocessors, the optimal fixed fraction is $80 \%$ of atoms and the automatic load balancing performance is within $13 \%$ of the fixed case. The results for rhodopsin are illustrated in Figure 1.

\subsection{Polymer blend}

For the second case, we chose a workload being used to study thermodynamic, transport, and rheological properties of hydrocarbon mixtures (typically 30-carbon atoms long) used as lubricants (C30 workload). This simulation uses 165600 atoms modeled using the CHARMM force field in a periodic box with an inner cutoff of $11 \AA$, an outer cutoff of $13 \AA$, and a neighbor list skin distance of $2 \AA$. Long range electrostatics were calculated using particle-particle particle-mesh with an accuracy of $1 e-4$. The simulation is sampled from the isothermalisobaric (NPT) ensemble at $313 \mathrm{~K}$ in a triclinic box that is deformed throughout the simulation. The performance measurements were for 400 timesteps with I/O including a single configuration dump. Again, a 10 step warm-up run was used before measurements.

The optimizations presented here resulted in a speedup of $1.45 \mathrm{X}$ when running on only the CPUs for the C30 workload.

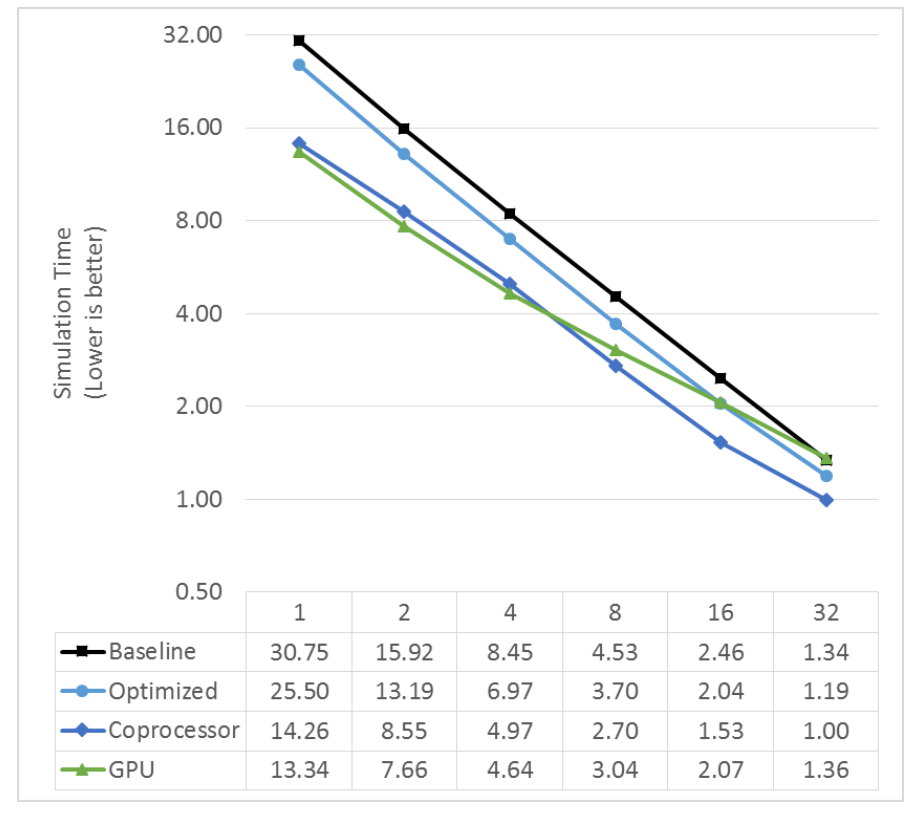

Figure 1: Simulation times with LAMMPS for the rhodopsin benchmark. Baseline is the best performance on CPUs with LAMMPS before optimizations. Coprocessor is simulation with offload to an Intel ${ }^{\circledR}$ Xeon Phi ${ }^{\mathrm{TM}} \mathrm{SE} 10 \mathrm{P}$ coprocessor. GPU is simulation with offload to an NVIDIA Tesla K20m GPU. Optimized is simulation with the routines optimized for the coprocessor, but using only the CPUs.

Offload to GPU resulted in a speedup of $2.15 \mathrm{X}$ on a single node and 1.25X on 32 nodes (about 300 atoms per CPU core). For both node counts, offload of a fixed fraction to Intel ${ }^{\circledR}$ Xeon Phi ${ }^{\mathrm{TM}}$ coprocessors was similar with a $2.33 \mathrm{X}$ speedup on 1 node and a $1.31 \mathrm{X}$ speedup on 32 nodes. Again, offload including ghost atoms was slightly faster with an offload of $60 \%$ of atoms performing best on 1 node, increasing to $80 \%$ at 32 nodes where overlap of communication and computation is more significant. Automatic load-balancing provided performance that was typically greater than $95 \%$ of the best fixed fraction. Best performing runs offloading to the Intel ${ }^{\circledR}$ coprocessors used 16 MPI tasks or 8 MPI tasks with 1-2 OpenMP threads. The results are illustrated in Figure 2.

\subsection{Organic photovoltaic molecules}

Developing a theoretical understanding of the factors influencing active-layer blend morphology is essential in the manufacture of efficient organic photovoltaics (OPV) [14]. An extension of a recent large-scale coarse-grained molecular dynamics 


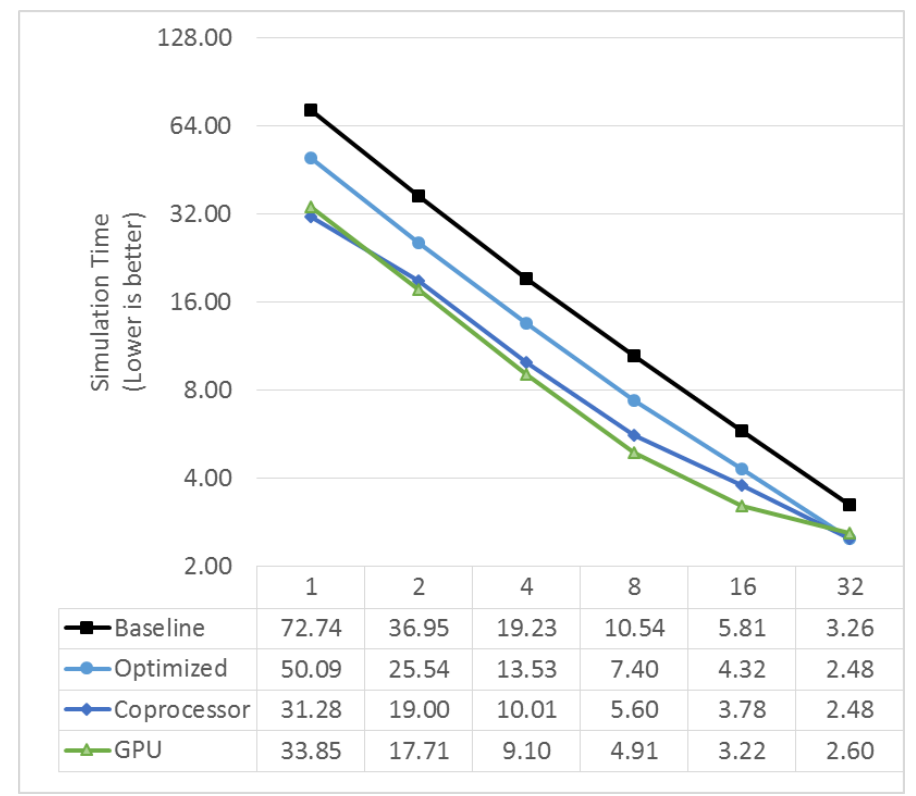

Figure 2: Simulation times with LAMMPS for the C30 simulation. Baseline is the best performance on CPUs with LAMMPS before optimizations. Coprocessor is simulation with offload to an Intel ${ }^{\circledR}$ Xeon Phi ${ }^{\mathrm{TM}} \mathrm{SE} 10 \mathrm{P}$ coprocessor. $G P U$ is simulation with offload to an NVIDIA Tesla K20m GPU. Optimized is simulation with the routines optimized for the coprocessor, but using only the CPUs.

of P3HT/PCBM blend[15] indicates that the addition of low molecular weight P3HT polymer chains, as a third component of the blend, can be used to alter the morphology. Intuitively it is expected that shorter chains should be more mobile hence the system kinetics should be accelerated and equilibrium morphology is attained at a faster rate. Unexpectedly however, short chains are found to efficiently migrate to the P3HT/PCBM interface. To understand better the results of the coarse-grained molecular dynamics simulation and model the P3HT/PCBM blend interface, all-atom simulations of low molecular weight P3HT in contact with PCBM are being performed. The aim is to investigate the sharpness of the donor/acceptor interface and orientation of thiophene rings at said interface.

In these all-atom simulations, the Generalized Amber ForceField (GAFF)[16] is used for both P3HT and PCBM. The description of a molecule in GAFF includes long-range coulomb and short-range van der Waals interactions, harmonic bonds, harmonic angles, and harmonic dihedrals. The mathematical description of the force-field potential used is similar to a validated P3HT-PCBM atomistic force-field [17]. P3HT oligomers, consisting of 9 monomers, are arranged in a slab configuration with P3HT located at the top and PCBM at the bottom portion of the simulation box. There are 2160 P3HT chains and 14480 PCBM molecules giving a total of 1,777,520 atoms in a $20.8 \times 20.8 \times 118 \AA^{3}$ simulation box. To benchmark performance of the simulations on different hardware, the simulation was run for $1.5 \mathrm{ps}$ in an isothermal-isobaric (NPT) ensemble utilizing a Nose-Hoover thermostat and barostat (423 $\mathrm{K}, 1 \mathrm{~atm}$ ) with an integration timestep of $1 \mathrm{fs}$ where the coordinates of the atoms and image snapshots are written to output files every $0.5 \mathrm{ps}$.

For the OPV simulation, the optimizations presented gave a $1.15 \mathrm{X}$ speedup when compared to the baseline code. Memory usage limitations required at least 4 nodes for GPU acceleration which resulted in a speedup of $2.08 \mathrm{X}$. At 32 nodes, the speedup was $1.38 \mathrm{X}$. Performance with offload to Intel ${ }^{\circledR}$ Xeon $\mathrm{Phi}^{\mathrm{TM}}$ coprocessors was similar with speedups ranging from $2.18 \mathrm{X}$ on 2 nodes to $1.4 \mathrm{X}$ on 64 nodes (Figure 3 ). Offload with ghost atoms was faster with the best fixed offload fractions ranging from $0.8 \mathrm{X}$ to $0.95 \mathrm{X}$. Automatic load balancing gave results that were typically within $90 \%$ of the performance of the best fixed fraction.

\subsection{Coarse-grained liquid crystal molecules}

For the final workload, we evaluated a liquid crystal benchmark based on previous materials simulations [18, 19] using the Gay-Berne potential [8]. Each liquid-crystal molecule is treated as a single ellipsoidal particle. The computational cost per atom (or particle in the coarse-grained case) of many potentials developed for materials science has grown significantly over time [20] and these models have perhaps the greatest potential improvements from vectorization and offload. In this benchmark, the simulation is comprised by $524 \mathrm{k}$ biaxial ellipsoidal liquid crystal mesogens with an aspect ratio of 2:1.5:1 and a mass of 1.5 (reduced units). The simulation is sampled from the microcanonical ensemble for 50 time steps, following a 10 time step warm-up. The short-range cutoff is 4.0 with a neighbor list 


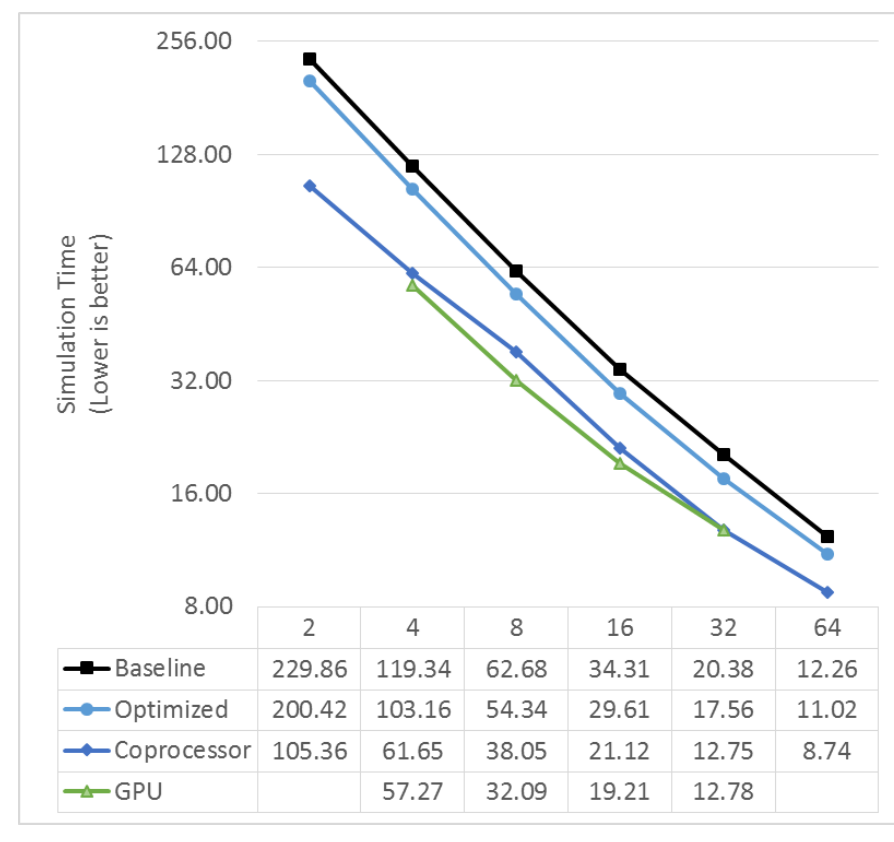

Figure 3: Simulation times with LAMMPS for the OPV simulation. Baseline is the best performance on CPUs with LAMMPS before optimizations. Coprocessor is simulation with offload to an Intel ${ }^{\circledR}$ Xeon Phi ${ }^{\mathrm{TM}} \mathrm{SE} 10 \mathrm{P}$ coprocessor. $G P U$ is simulation with offload to an NVIDIA Tesla K20m GPU. Optimized is simulation with the routines optimized for the coprocessor, but using only the CPUs.

skin of 0.8. This benchmark simulation is also available in the LAMMPS distribution.

The optimizations presented here resulted in a $4.68 \mathrm{X}$ speedup compared to the baseline code when running only on CPUs. Offload to GPU resulted in a speedup of 7.64X using 14 MPI tasks per node. Offload to Intel ${ }^{\circledR}$ Xeon Phi ${ }^{\mathrm{TM}}$ coprocessors resulted in a speedup of $7.12 \mathrm{X}$ with $16 \mathrm{MPI}$ tasks offloading $55 \%$ of the atoms without any ghost atoms. In contrast to the other benchmarks presented, offload including ghost atoms was slower. Performance using automatic load balancing resulted in a simulation rate that was $95 \%$ of the best time obtained with a fixed offload fraction. These results are illustrated in Figure 4 along with the speedups from the other workloads on 1 or 4 nodes.

\subsection{Correctness}

We have tested the software using a series of 7680 regression tests that compare the results to the baseline LAMMPS using different workloads and options. For the results presented

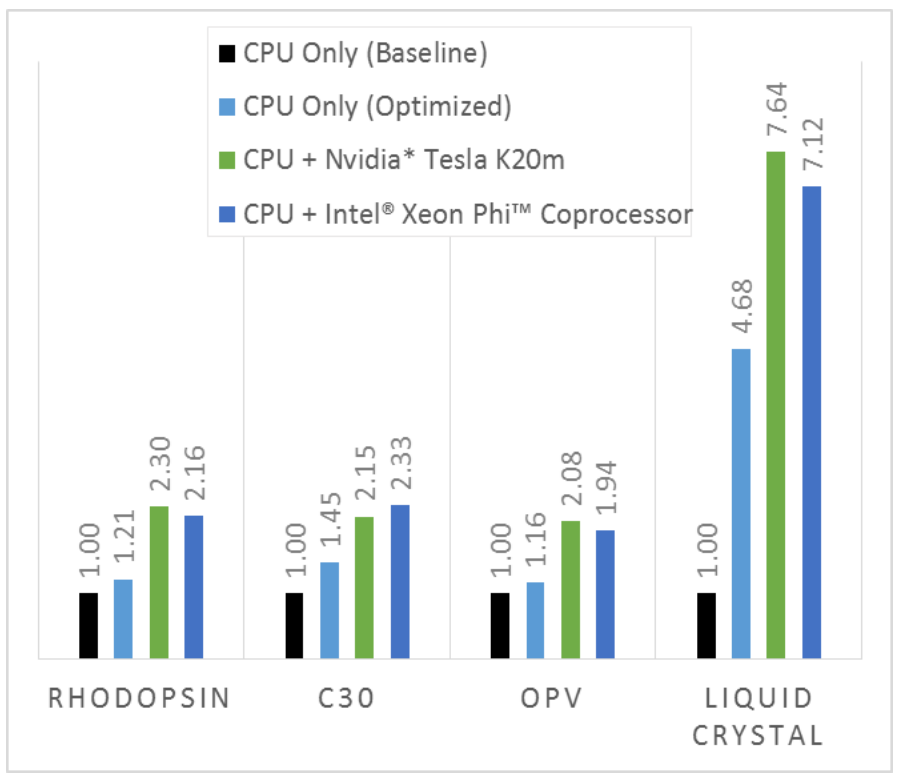

Figure 4: Speedups obtained with offload to an NVIDIA Tesla K20m GPU or an Intel ${ }^{\circledR}$ Xeon $\mathrm{Phi}^{\mathrm{TM}}$ coprocessor on Stampede for several workloads on 1 node (Rhodopsin, C30, Liquid Crystal) or 4 nodes (OPV). "Optimized" is the speedup from optimizations using the same code run only on the CPUs.

here, all output files were checked to assure that thermodynamic quantities including energy, temperature, and pressure were identical to the baseline code in the most significant digits. If we take the average initial and final values for the different MPI/OpenMP runs with the baseline code as the range, all final results with the Intel package were within $0.08 \%$ of the final baseline average with the exception of the polymer blend. Because the polymer blend runs were short and the initial configuration was not equilibrated, there were significant fluctuations in final results within the baseline code depending on the MPI/OpenMP configuration. However, final values for the Intel package were comparable with mean values differing by no more than $18 \%$ of the standard deviation observed in the baseline final values. Energy conservation was also tested to verify correctness for the simulation models. This is shown in Figure 5 for the liquid crystal benchmark modified to run 30000 timesteps in the microcanonical ensemble. Although significant deviations are not seen when using single precision in the plot, the authors do not recommend running in single precision without significant validation for the workload. 


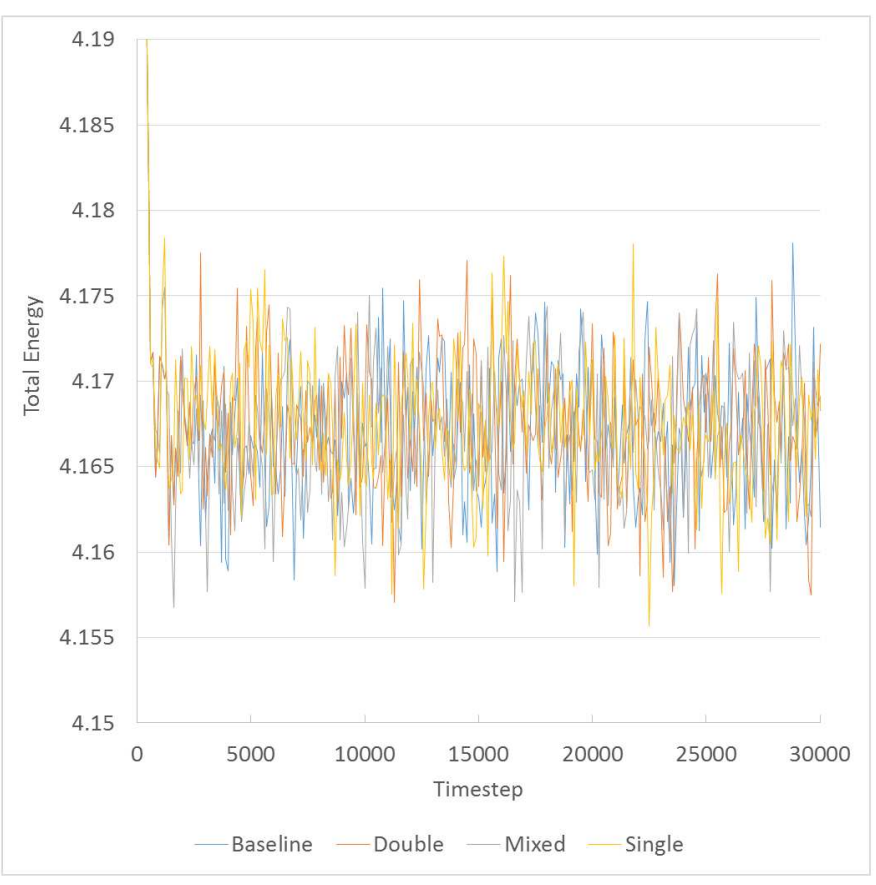

Figure 5: Energy conservation for the liquid crystal benchmark. Results are shown for the baseline code and the Intel package using single, mixed, and double precision modes.

\section{Discussion}

For the workloads presented here, we have found that optimizations to support mixed precision and vectorization, along with LEO directives, resulted in performance on hybrid CPU/Intel ${ }^{\circledR}$ Xeon Phi ${ }^{\mathrm{TM}}$ coprocessor nodes that was similar to the existing GPU-acceleration algorithms in LAMMPS. This includes a standard LAMMPS benchmark, but more importantly, production simulations including statistics and $\mathrm{I} / \mathrm{O}$ that are representative of actual runs. The latter is a more difficult problem for optimization than the use of proxy-MD kernels (or simple benchmarks that only utilize proxy-kernels). For the simulations currently being used to study the active layers of organic solar cells and thermodynamic properties of hydrocarbon lubricants, we can now achieve simulation rates over $2 \mathrm{X}$ what was previously possible.

The option for excluding ghost atoms from offload calculations resulted in better performance for the liquid crystal simulation, but worse in others. This option can allow for better overlap of computation with communication, in trade for shorter neighbor lists evaluated on the coprocessor (and therefore potentially reduced benefit from vectorization). Currently, in LAMMPS, the option is selected automatically based on the potential energy model used; users can change the default for fine tuning if desired. Automatic load balancing typically gave performance that was within $10 \%$ of the best performance obtained using a fixed fraction. Part of the decrease in performance was likely due to the exclusion of data transfer times from the calculations. We hope that new versions of the compilers will support timing that includes data transfer in order to improve load balancing performance.

In some cases, GPU performance was slightly better and in others, Intel ${ }^{\circledR}$ Xeon Phi ${ }^{\mathrm{TM}}$ performance was better. When using more recent processors, Intel ${ }^{\circledR} \mathrm{Xeon}^{\circledR} \mathrm{E} 5-2697 \mathrm{v} 2$ or E52697 v3 with Intel ${ }^{\circledR}$ Xeon Phi ${ }^{\mathrm{TM}} 7120$ or Nvidia Tesla K40c, we find that performance shifts more in favor of offload to Intel ${ }^{\circledR}$ coprocessors [data not shown]. However, future Nvidia Tesla GPUs might offer improved GPU-acceleration in LAMMPS.

The main differentiating factor we stress is that the directivebased programming model used for the Intel ${ }^{\circledR}$ coprocessors allows the same routine to efficiently execute on both the CPU and the coprocessor. This is relevant because of the prevalence of $\mathrm{x} 86$-based processors in HPC machines, the fact that many HPC codes using GPU acceleration (including LAMMPS) rely on the CPUs for a significant fraction of the simulation, the necessity for popular HPC codes to continue to support $\mathrm{x} 86$ in order to be useable at the majority of HPC centers, and the upcoming release of Intel ${ }^{\circledR}$ many-core processors that are self-hosted where no coprocessor is present. Although directive-based models such as OpenACC can be used for GPU-acceleration, the fact that the best-performing algorithms in LAMMPS are substantially different for the GPU and x86 would still result in different code for using the two architectures, making future porting and debugging efforts more difficult.

Although the code optimized for Intel ${ }^{\circledR}$ Xeon $\mathrm{Phi}^{\mathrm{TM}}$ coprocessors provided advantages for the CPUs, running unmodified code on the coprocessors would be much 
slower and this issue is commonly encountered, e.g. when a legacy code is optimized incrementally for new hardware. The penalties for data movement and unvectorized code are amplified on many-core processors. However, optimizations to address these issues are important for future power-efficient processors. Regardless of coprocessors and GPU accelerators, vector widths and core counts have been increasing on server processors for some time. In the case of the optimizations for GPU acceleration, addressing these issues for the CPU was not important; on most current configurations, offload of the entire neighbor and force kernels performs best. It is clear from the results presented here, however, that this approach leaves CPU performance on the table - in extreme cases the optimizations led to improvements over $4.5 \mathrm{X}$ for the entire simulation on the CPUs (the coarse-grained Gay-Berne model discussed in the previous section).

In our experience ${ }^{1}$, the code optimizations discussed here required substantially less effort for the Intel ${ }^{\circledR}$ Xeon $\mathrm{Phi}^{\mathrm{TM}}$ coprocessor when compared to the GPU. It is difficult to provide quantitative evidence for this, however, we note in this effort the same routines can be run on both CPUs and the coprocessors, they can be compiled with any $\mathrm{C}++$ compliant compiler, and they will run efficiently on machines without coprocessors. For any code, this software development model should reduce future maintenance and porting efforts for the developers.

The optimizations described here are available in LAMMPS as an optional package. This approach can give scientists access to improved performance now, while still allowing the developers to experiment with code modernization strategies to improve future performance and converge on models and algorithms that will perform best and can eventually be adopted as the default in LAMMPS.

\footnotetext{
${ }^{1}$ The author WMB was also the lead developer of the GPU package used in LAMMPS for the last 4 years $[1,2]$
}

\section{Acknowledgements}

All of the code described in this paper is available in the open-source LAMMPS software package, available at http://lammps.sandia.gov/ or by contacting the authors. The authors acknowledge the Texas Advanced Computing Center (TACC) at The University of Texas at Austin for providing HPC resources that have contributed to the research results reported within this paper. URL: http://www.tacc.utexas.edu. Sandia is a multipurpose laboratory operated by Sandia Corporation, a Lockheed-Martin Co., for the U.S. Department of Energy under Contract No. DE-AC04-94AL85000. J.-M.Y.C. acknowledges support from the Office of Advanced Scientific Computing Research, Office of Science, U.S. Department of Energy under Contract No. DE-AC05-00OR22725 with UT-Battelle, LLC. This research used resources of the Leadership Computing Facility at Oak Ridge National Laboratory, which is supported by the Office of Science of the U.S. Department of Energy under Contract No. DE-AC05-00OR22725 with UT-Battelle, LLC. The authors thank Shell Lubricants Research team in Houston for providing the hydrocarbon structures.

\section{References}

[1] W. M. Brown, P. Wang, S. J. Plimpton, A. N. Tharrington, Computer Physics Communications 182 (2011) 898-911.

[2] W. M. Brown, A. Kohlmeyer, S. J. Plimpton, A. N. Tharrington, Comp. Phys. Comm. 183 (2012) 449-459.

[3] W. M. Brown, T. D. Nguyen, M. Fuentes-Cabrera, J. D. Fowlkes, P. D. Rack, M. Berger, A. S. Bland, Procedia Comput. Sci. 9 (2012) 186-195.

[4] W. M. Brown, M. Yamada, Computer Physics Communications 184 (2013) 2785-2793.

[5] S. Plimpton, Journal of Computational Physics 117 (1995) 1-19.

[6] S. J. Pennycook, C. J. Hughes, M. Smelyanskiy, S. A. Jarvis, in: Parallel $\&$ Distributed Processing (IPDPS), 2013 IEEE 27th International Symposium on, IEEE, pp. 1085-1097.

[7] S. Páll, B. Hess, Computer Physics Communications 184 (2013) 26412650 .

[8] R. Berardi, C. Fava, C. Zannoni, Chem. Phys. Lett. 236 (1995) 462-468.

[9] Stampede, Top500 Supercomputer Sites, Stampede http://www.top500.org/system/177931, (accessed Oct 14, 2014).

[10] A. D. MacKerell, D. Bashford, M. Bellott, R. Dunbrack, J. Evanseck, M. J. Field, S. Fischer, J. Gao, H. Guo, S. a. Ha, et al., The Journal of Physical Chemistry B 102 (1998) 3586-3616. 
[11] R. W. Hockney, S. P. Goel, J. W. Eastwood, Journal of Computational Physics 14 (1974) 148-158.

[12] J.-P. Ryckaert, G. Ciccotti, H. J. Berendsen, Journal of Computational Physics 23 (1977) 327-341.

[13] P. S. Crozier, M. J. Stevens, L. R. Forrest, T. B. Woolf, Journal of molecular biology 333 (2003) 493-514.

[14] F. Liu, Y. Gu, J. W. Jung, W. H. Jo, T. P. Russell, Journal of Polymer Science Part B: Polymer Physics 50 (2012) 1018-1044.

[15] J.-M. Y. Carrillo, R. Kumar, M. Goswami, B. G. Sumpter, W. M. Brown, Physical Chemistry Chemical Physics 15 (2013) 17873-17882.

[16] J. Wang, R. M. Wolf, J. W. Caldwell, P. A. Kollman, D. A. Case, Journal of computational chemistry 25 (2004) 1157-1174.

[17] T. To, S. Adams, Physical Chemistry Chemical Physics 16 (2014) 46534663.

[18] W. M. Brown, M. K. Petersen, S. J. Plimpton, G. S. Grest, Journal of Chemical Phyics 130 (2009) 044901.

[19] T. D. Nguyen, J.-M. Y. Carrillo, M. A. Matheson, W. M. Brown, Nanoscale 6 (2014) 3083-3096.

[20] S. J. Plimpton, A. P. Thompson, MRS Bull 37 (2012) 513-521. 